\title{
Establishment of a ferroptosis-related gene signature for prognosis in lung adenocarcinoma patients
}

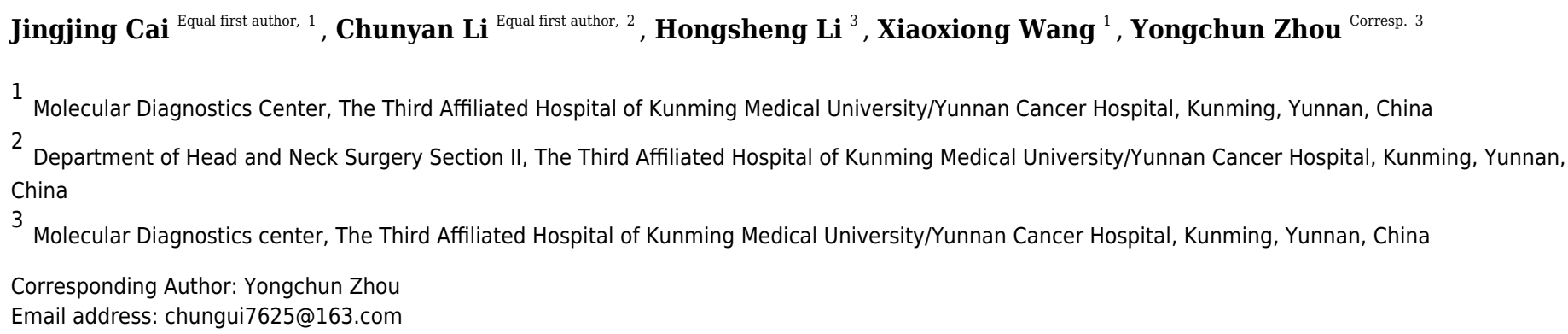

Objective: Lung cancer is the most common malignancy worldwide and exhibits both high morbidity and mortality. In recent years, scientists have made substantial breakthroughs in the early diagnosis and treatment of lung adenocarcinoma (LUAD), however, patient prognosis still shows vast individual differences. In this study, bioinformatics methods were used to identify and analyze ferroptosis-related genes to establish an effective signature for predicting prognosis in LUAD patients. Methods: The gene expression profiles of LUAD patients with complete clinical and follow-up information were downloaded from two public databases, univariate Cox regression and multivariate Cox regression analysis were used to obtain ferroptosis-related genes for constructing the prognos tic risk model, AUC and calibration plot were used to evaluate the predictive accuracy of the FRGS and nomogram. Results: A total of 74 ferroptosis-related differentially expressed genes (DEGs) were identi fied between LUAD and normal tissues from The Cancer Genome Atlas (TCGA) database. A five- gene panel for prediction of LUAD prognosis was established by multivariate regression and was veri fied using the GSE68465 cohort from the Gene Expression Omnibus (GEO) database. Patients were divided into two different risk groups according to the median risk score of the five genes. Based on Kaplan-Meier (KM) analysis, the OS rate of the high-risk group was markedly worse than that of the low-risk group. We also found that risk score was an independent prognostic indicator. The receiver operating characteristic (ROC) curve showed that the proposed model had good prediction ability. Gene Ontology (GO) and Kyoto Encyclopedia of Genes and Genomes (KEGG) functional analyses indicated that risk score was prominently enriched in ferroptosis processes. Moreover, at the score of immune-associated gene sets, significant differences were found between the two risk groups.Conclusions: This study demonstrated that ferroptosisrelated gene signatures can be used as a potential predictor for the prognosis of LUAD, thus providing a novel strategv for individualized treatment in LUAD patients. 
1 Establishment of a ferroptosis-related gene signature for prognosis in lung

2

8 Corresponding Author: Yongchun Zhou

9 Email address: chungui7625@163.com

12 Abstract

13 Objective:

14 Lung cancer is the most common malignancy worldwide and exhibits both high morbidity and multivariate Cox regression analysis were used to obtain ferroptosis-related genes for mortality. In recent years, scientists have made substantial breakthroughs in the early diagnosis and treatment of lung adenocarcinoma (LUAD), however, patient prognosis still shows vast individual differences. In this study, bioinformatics methods were used to identify and analyze ferroptosis-related genes to establish an effective signature for predicting prognosis in LUAD patients.

Methods :

1 The gene expression profiles of LUAD patients with complete clinical and follow-up information were downloaded from two public databases, univariate Cox regression and 24 constructing the prognostic risk model, AUC and calibration plot were used to evaluate the 25 predictive accuracy of the FRGS and nomogram.

26 Results: 
27 A total of 74 ferroptosis-related differentially expressed genes (DEGs) were identified 28 between LUAD and normal tissues from The Cancer Genome Atlas (TCGA) database. A five29 gene panel for prediction of LUAD prognosis was established by multivariate regression and was 30 verified using the GSE68465 cohort from the Gene Expression Omnibus (GEO) database.

31 Patients were divided into two different risk groups according to the median risk score of the five 32 genes. Based on Kaplan-Meier (KM) analysis, the OS rate of the high-risk group was markedly 33 worse than that of the low-risk group. We also found that risk score was an independent 34 prognostic indicator. The receiver operating characteristic (ROC) curve showed that the 35 proposed model had good prediction ability. Gene Ontology (GO) and Kyoto Encyclopedia of 36 Genes and Genomes (KEGG) functional analyses indicated that risk score was prominently 37 enriched in ferroptosis processes. Moreover, at the score of immune-associated gene sets, 38 significant differences were found between the two risk groups.

39 Conclusions:

40 This study demonstrated that ferroptosis-related gene signatures can be used as a potential 41 predictor for the prognosis of LUAD, thus providing a novel strategy for individualized 42 treatment in LUAD patients.

43 Keywords: lung adenocarcinoma, ferroptosis, gene signature, prognosis, immune status

44 Introduction

45 The incidence and mortality of lung cancer rank first in the world (Siegel, R.L., et al, 2020). 46 Among all lung cancer subtypes, lung adenocarcinoma (LUAD) accounts for the highest 47 proportion $(\sim 40 \%$ ) (Testa, U., et al, 2018). At present, LUAD treatments include surgery, 48 radiotherapy, and chemotherapy, as well as targeted therapy and immunotherapy (Eberhardt, W.E., 49 et al,2015). However, regardless of conventional or novel combination therapy, there appears to 
50 be no significant improvement in patient prognosis, metastasis risk, or recurrence rate, and five51 year survival remains at only $15 \%$ (Chen, H., et al.,2019). Therefore, it is crucial to find innovative

52 prognostic models to provide better diagnosis and treatment strategies for LUAD patients.

53 Scientists have recently discovered a novel type of programmed cell death that differs from 54 apoptosis and cell necrosis, called ferroptosis, which depends on iron ions and reactive oxygen

species (ROS) to induce lipid peroxide accumulation (Latunde-Dada, G.O.,2017; Stockwell, B.R., et al,2017; Conrad, M., et al, 2018). An increasing body of evidence suggests that ferroptosis is involved in the initiation, progression, and suppression of cancer (Fearnhead, H.O., et al, 2017). Thus, induction of ferroptosis may be an emerging target for the treatment of malignant tumors (Liang, C., et al, 2019; Hassannia, B.,et al,2019), and polyunsaturated fatty acid (PUFA) in phospholipids, redox active iron, and lipid peroxidation (LPO) repair defects, may determine the susceptibility of cancer cells to ferroptosis. Current studies have shown that ferroptosis mainly involves two pathways: GSH / GPX4 pathway and FSP1 / CoQ / NADPH pathway (Dixon S J., et al, 2012). P53 is the most closely related tumor suppressor gene. It can not only induce apoptosis, but also induce ferroptosis. P53 can inhibit the absorption of cystine by systemxc by inhibiting the transcription of SLC7A11 (Wang S J., et al, 2016), resulting in the inhibition of GSH / GPx4 pathway, the reduction of cell antioxidant capacity and the occurrence of ferroptosis. Jiang et al. (Jiang L., et al, 2015) confirmed that SLC7A11 is a new regulatory target of p53 gene. Some genes, such as SLC7A11 (Ji, X., et al,2018), SLC3A2 (Huang, Y., et al,2005), and STYK1 (Lai, Y., et al,2019), are overexpressed in lung cancer cells and mediate the inhibition of ferroptosis. In addition, some lung cancer drugs have been shown to induce ferroptosis. For example, cisplatin is reported to be an inducer of ferroptosis in non-small-cell lung cancer (NSCLC) A549 cells (Guo, J., et al,2018) and cisplatin and erastin (a type I ferroptosis inducer 
73 (FIN)) in combination exhibit synergistic effects on anti-lung cancer cell activity. Sorafenib has

74 also been found to induce ferroptosis in the lung cancer cell line NCI-H460 (Lachaier, E., et al, 75 2014). In addition, previous studies have found that GPX4 had high activity in cells with 76 epithelial mesenchymal transition related gene expression. When using first-line chemotherapy 77 drugs to treat melanoma, breast cancer and lung cancer cell lines, the remaining drug-resistant 78 cancer cells were found to have stem cell like characteristics, mesenchymal like gene expression 79 characteristics and GPX4 dependent characteristics (Hangauer M J., 2017). However, there are 80 almost no studies on ferroptosis-related genes and LUAD prognosis. In this research, we 81 explored the prognostic role of ferroptosis-related genes in LUAD.

82 Materials \& Methods

83 Databases

84 We used the NCI Genomic Data Commons (https://portal.gdc.cancer.gov) for online analysis and 85 visualization of genomic data from The Cancer Genome Atlas (TCGA) to obtain raw data. We 86 downloaded the TCGA-LUAD Htseq_counts.tsv dataset, which contains 528 tumor samples and 8757 normal samples, and downloaded related phenotype information (e.g., age, sex, and TNM 88 stage) and corresponding survival information (e.g., survival status and time to latest follow-up).

89 We searched the LUAD gene expression dataset from the Gene Expression Omnibus (GEO; 90 http://www.ncbi.nlm.nih.gov/geo/) database and collected a cohort based on the GPL96 91 platform, resulting in 441 cancer patients with complete clinical information.

92 Collection of ferroptosis-related data

93 FerrDb (http://www.zhounan.org/ferrdb) (Zhou, N. and J. Bao,2020) is a manually collected 94 and curated database for the study of markers and regulators of ferroptosis and the association of 95 ferroptosis disease. At present, ferroptosis-related genes (259) have been found and reported in 
96 the literature (Supplementary Table S1).

97 Identification of differentially expressed genes (DEGs)

98 The DEGs were obtained using the "limma" software package(Ritchie ME, et al,2015) and

99 those DEGs with $\log 2 \mathrm{FC} \mid \geq 1$ and $P a d j<0.05$ were included in subsequent analyses.

100 Constructing and validating a prognostic ferroptosis-related gene signature

101 The ferroptosis-related genes associated with prognosis in LUAD patients were identified 102 using univariate Cox regression analysis. Genes with an adjusted $P$-value of $<0.05$ were 103 included for further analysis. The STRING online database (STRING; http://string-db.org) 104 (v11.0) (Szklarczyk, D., et al, 2019) was used to analyze the interactions among genes with 105 significant differences in univariate analysis, and a protein-protein interaction (PPI) network was 106 constructed. Using Cytoscape (v3.7.1) (Smoot, M.E., et al, 2011) to further visualize the DEGs, the 107 top ten genes were screened by the degree method using the cytoHubba plug-in. Multivariate 108 Cox regression analysis was used to obtain the gene panel for constructing the prognostic risk 109 model, which was determined as risk score $=\sum($ Coefi $\times$ Expi $)$. According to the median 110 valuescalculated using the R software packages "survminer" and "survival", the tumor samples 111 were divided into high-risk or low-risk groups. Kaplan-Meier (KM) survival was further plotted 112 using the "survival" package (Ranstam J, Cook JA, 2017). A time-dependent receiver operating 113 characteristic (ROC) curve drawn with the R package "survivalROC" was used to test the 114 accuracy of the model prediction (Szklarczyk, D., et al, 2019)

\section{Nomogram generation}

116 The R package "RMS" was used to draw a compound nomogram based on risk scores and 117 clinicopathological characteristics, and consistency between the predicted and actual results was 118 evaluated using a calibration curve. 
119 Biological pathway of ferroptosis-related prognostic genes

120 Gene Ontology (GO) and Kyoto Encyclopedia of Genes and Genomes (KEGG) enrichment

121 analyses (Ashburner M., et al, 2000)of DEGs in the two risk groups were performed to determine

122 their main biological functions. The "clusterProfiler" package with $\mathrm{R}$ language was used to

123 generate corresponding files.Single sample gene set enrichment analysis (ssGSEA) and the "gsva"

124 package (Hänzelmann S., et al, 2013) in R were used for scoring each sample in the two risk

125 groups based on 29 immune-related genes (Supplementary Table S2) sets to explore the

126 correlations among different risk groups and immune status.

127 Statistical analysis

128 Univariate Cox regression, multivariate Cox regression, wilcoxon testand log rank tests 129 were used in this study. Statistical analysis and related figures were generated using R software 130 (v3.5.3) (Diboun, I., et al, 2006). In this study, $P$ adj $<0.05$ was regarded as statistically 131 significant.

132 Results

133 Patient characteristics and DEGs

134 Our study flow chart is shown in Fig. 1. In total, there were 528 LUAD patients in the 135 TCGA dataset and 441 LUAD patients in the GSE68465 dataset. Their clinical characteristics 136 are shown in Table 1.

137 We obtained 5438 DEGs in the TCGA database according to $|\log 2 \mathrm{FC}| \geq 1$ and $\operatorname{Padj}<0.05$.

138 We used Venn online analysis to identify and visualize overlapping DEGs in the two databases 139 and downloaded the Venn diagram (Fig. 2a). The heat map and volcano map (Fig. 2b, c) showed 14048 up-regulated genes and 26 down-regulated genes. The list of DEGs is shown in Supplement 3. 141 PPI analysis of DEGs 
142 Using univariate Cox regression analysis, we identified 17 genes related to prognosis in

143 LUAD patients (Fig. 3a), the correlations of which are shown in Fig. 3b. We then imported the

144 above DEGs into the STRING database, and a PPI network was obtained after the lowest

145 confidence was set to 0.015 and genes without interactions were removed (Fig. 3c).

146 Subsequently, we used Cytoscape and cytoHubba to sketch the top 10 genes. SLC7A11,

147 SLC7A11, and GDF15 were determined to be the top hub genes (Fig. 3d).

148 Construction and validation of a prognostic model in TCGA and GEO cohorts

149 We obtained a five-gene panel as a prognostic signature using the multivariate Cox 150 regression model. The forest plot of the five-gene panel is shown in Fig. 4a. The five screened 151 genes were Arachidonate 15-Lipoxygenase (ALOX15), DNA Damage Inducible Transcript 4 152 (DDIT4), Hepatocyte Nuclear Factor 4 Alpha (HNF4A), Interleukin 33 (IL33), and Growth 153 Differentiation Factor 15 (GDF15). Among them, DDIT4 and HNF4A were highly expressed in 154 tumor tissues, whereas ALOX15, IL33, and GDF15 were lowly expressed (Table 2). Risk score $155=(-0.061165052 \times$ expression ALOX15) $+(0.169594473 \times$ expression DDIT4 $)+(0.072356312$ $156 \times$ expression HNF4A $)+(-0.131023567 \times$ expression IL33) $+(-0.107882858 \times$ expression 157 GDF15). Therefore, the risk scores of LUAD patients in the two databases were calculated 158 according to the Cox regression model (composed of five genes).

159 Patients were dichotomized into low-risk score $(\mathrm{n}=251)$ and high-risk score groups $(\mathrm{n}=$ 160 250) based on the median cut-off value (Fig. 5a). Compared with the low-risk group, the high161 risk group showed a higher risk of death (Fig. 5c). Based on three-dimensional (3D) principal 162 component analysis (PCA), the prognosis model clearly distinguished the LUAD tumor samples 163 of the two risk groups (Fig. 5e). Furthermore, KM curve analysis showed that the OS of the high164 risk group was significantly worse than that of the low-risk group (Fig. $5 \mathrm{~g}, P<0.001$ ). The 
165 clinical correlation heat map of the two risk groups is shown in Fig. 5i. A time-dependent ROC

166 curve was used to test the predictive performance of the OS risk score composed of the five-gene

167 panel. Results showed that the AUCs of one-year, two-years, and three-years were $0.704,0.668$, 168 and 0.693, respectively (Fig. 5k).

169 We also observed similar survival curves, survival statuses, risk scores, patient distributions, 170 and clinical prognostic characteristics in the validated GSE68465 dataset (Fig. 5b, d, f, h, j).

171 When we performed 1-, 2-, and 3-year ROC curve analyses to assess the predictive capacity of 172 the prognostic five-gene signature, we found the AUCs for 1-, 2-, and 3-year OS predictions 173 were $0.653,0.644$, and 0.617 , respectively(Fig. 51).

174 Nomogram establishment

175 Based on the two cohorts, we constructed a nomogram using clinicopathological 176 characteristics (age, sex, TNM stage, grade) and risk scores, respectively, and calculated the total 177 score of each patient to predict the one-year, two-year, and three-year survival rates of the 178 LUAD patients (Fig. 6a). Further calibration curves showed that the third year OS predicted by 179 the nomogram was in good agreement with actual OS (Fig. 6b).

180 Assessment of independent prognostic value of risk score

181 To evaluate the relationship between risk score and prognosis of the five-gene model, we 182 used risk score as an index and clinicopathological characteristics of LUAD patients for 183 univariate and multivariate Cox regression analyses. Available variables of the TCGA cohort 184 included age, sex, TMN stage, and risk score. The GSE68465 cohort included age, sex, TN stage, 185 and risk score. In the TCGA cohort, the risk score was determined as an independent prognostic 186 factor for $\mathrm{OS}(\mathrm{HR}=1.809,95 \% \mathrm{CI}=1.292-2.535, P<0.001 ; \mathrm{HR}=1.496,95 \% \mathrm{CI}=1.056-$ 
187 2.121, $P=0.023$ ) (Fig. 7a). The GSE68465 cohort results were consistent (HR = 1.625, 95\% CI

$188=1.254-2.105, P<0.001 ; \mathrm{HR}=1.425,95 \% \mathrm{CI}=1.098-1.850, P=0.008)$ (Fig. 7b).

189 Functional enrichment analysis in TCGA and GEO cohorts

190 We used the $\mathrm{R}$ language clusterProfiler package to analyze enrichment in biological 191 functions (GO) and KEGG pathways for the 1285 DEGs in the high- and low-risk groups in the

192 TCGA cohort. In total, 40 important functional annotations $(P<0.05$, Fig. 8a) were enriched in 193 molecular functions, among which five were iron related $(P<0.05$, Fig. 8a), including a variety 194 of enzymes, gluconosyltransferase and oxidoreductase activity. Unfortunately, the 16 195 significantly enriched pathogenic KEGG pathways $(P<0.05$, Fig. 8 c) were not associated with 196 ferroptosis. In the GSE68465 validation cohort, multiple functional iron-related molecules were 197 enriched $(P<0.05$, Fig. 8b). In addition, one iron-related pathway, namely ferroptosis, was 198 enriched in the KEGG validation cohort $(P<0.05$, Fig. $8 \mathrm{~d})$.

199 We used ssGSEA to further score the samples from different risk groups in the TCGA and 200 GSE68465 cohorts. Differences in different immune cells, functions, and pathways were 201 detected between the two groups (Fig. 9a-b). The high-risk group in the TCGA cohort showed 202 lower scores in immune-related cells, such as mast cells, neutrophils, dendritic cells (DCs), and T 203 helper cells, with only natural killer (NK) cells showing higher scores (all adjusted $P<0.05$, Fig. 204 9a).Similar results were obtained for the GSE68465 cohort (all adjusted $P<0.05$, Fig. 9b).For 205 pathways, the high-risk group in the two cohorts showed lower scores for type II and type I IFN 206 responses (all adjusted $P<0.05$, Fig. 9b).

\section{Discussion}

208 While the incidence rate of lung cancer is no longer the top priority, the mortality rate is still 209 first among cancer deaths, accounting for more than 180000 cases [20].At present, LUAD is still 
210 the most common histological subtype of lung cancer, with high mortality among Asians, 211 females, and non-smoking patients (Chen, J., et al, 2020). Although LUAD treatment has made 212 great progress in recent years, most patients with LUAD still exhibit poor prognosis and a low 213 five-year survival rate due to tumor recurrence and metastasis. Therefore, a reliable prognostic 214 biomarker is crucial for evaluating and predicting prognosis in LUAD patients. Recently, 215 bioinformatics analyses have become an important screening tool in cancer research(Huang, 216 C.C.,et al,2019).

217 Most previous studies have focused on the relationship between ferroptosis and 218 tumorigenesis, development, proliferation, and invasion, with only one study by Liang et al. 219 (Liang, J.Y., et al,2020) exploring ferroptosis-related genes and survival rates in patients with 220 hepatocellular carcinoma (HCC). In this study, we used two independent databases and 221 constructed a ferroptosis-related gene panel to predict OS in patients with LUAD. Firstly, we performed univariate Cox regression analysis on 74 ferroptosis-related DEGs in LUAD patients 223 and found that 17 genes were excellent predictors of prognosis, including seven down-regulated and 10 up-regulated genes. Five ferroptosis-related genes (i.e., ALOX15, ddit4, HNF4A, IL33, and GDF15) were further screened by multivariate regression analysis. We then divided patients into high- and low-risk groups according to their median risk score. KM curve analysis showed that the high-risk group was associated with poorer survival compared with the low-risk group. An ROC curve was used to evaluate the prognostic reliability of this signature. The nomogram results indicated that the risk-score model may be an effective method to predict survival status in LUAD patients over different years. We applied GO and KEGG pathway enrichment analysis and found that risk score was significantly correlated with the biological functions and pathways

232 of ferroptosis. SsGSEA further showed that there were significant differences in the immune 
233 status of the DEGs between the high-risk group and low-risk group.

234 ALOX15 encodes a member of the lipoxygenase family of proteins, which can regulate 235 inflammation and immune responses. Recent studies have shown that ALOX15 is not only 236 involved in apoptosis, but also in autophagy and ferroptosis (Dixon, S.J., et al, 2012). ALOX15 is 237 involved in ferroptosis through multiple pathways (Stoyanovsky, D.A., et al, 2019), including 238 regulating the activation of Ras selective lethal small molecular 3 (RSL3) ( Probst, L., et al,2017), 239 reducing the activation of glutathione (GSH), and forming a complex with 240 phosphatidylethanolamine binding protein 1(PEBP1) (Wenzel, S.E., et al,2017). Mounting 241 evidence indicates that ALOX15 is down-regulated in many human cancers, including colorectal 242 (Shureiqi, I., et al, 2000), prostate (Tang, S., et al, 2002), breast (Jiang, W.G., et al, 2003) and lung 243 cancers(Gonzalez, A.L., et al,2004). Gonzalez et al. (Gonzalez, A.L., et al, 2004) also reported that 244 the expression of 15-LOX-2 is higher in better differentiated NSCLC and is negatively correlated 245 with tumor grade and tumor cell proliferation. However, whether ALOX15 participates in the 246 occurrence of NSCLC by targeting ferroptosis remains unclear. Previous studies have indicated 247 that the use of 12/15-LOX inhibitors or the silencing of ALOX15 expression can prevent cancer 248 cells (including Calu-1 human NSCLC) with RAS expression from cell death in erastin- and 249 RSL3-induced ferroptosis (Shintoku, R., et al, 2017).

250 DDIT4 is a stress-response protein whose main function is to inhibit mTOR under stressful 251 conditions.DDIT4 is considered an oncogene (Smith, E.R.,X.X. Xu;2009), and its overexpression 252 is significantly associated with poorer prognosis in tumor patients(Tirado-Hurtado, I., et al,2018). 253 Jin et al. (Jin, H.O., et al, 2019) reported that constitutive overexpression of DDIT4 can lead to 254 HSP27 and HSP70 induction and AKT activation. This mechanism is related to lung cancer cell 255 survival and IR resistance, indicating that DDIT4 may be a therapeutic target for lung cancer. 
GDF15 is a member of the transforming growth factor-beta superfamily, and its association

257

258

259

260

261

262

263

264

265

266

267

268

269

270

271

272

273

274

275

276

277

278

with cancer can depend on cell state and tumor environment. Recent study suggests that GDF15

is lowly expressed in NSCLC, and its down-regulation is associated with poor prognosis in such

patients (Lu, X., et al, 2018).GDF15 is also suggested to inhibit the growth and bone metastasis of LUAD A549 cells by targeting the TGF- $\beta /$ Smad signaling pathway (Duan, L., et al, 2019).However, GDF15 is up-regulated in some malignant tumors, such as gastric cancer in humans. For example, Chen et al. (Chen, L., et al, 2020) recently reported on the role of GDF15 in gastric cancer cell (MGC803) ferroptosis.GDF15 also plays an important role in erastin-induced ferroptosis by affecting the function of system Xc and regulating the expression of SLC7A11 (Chen, L., et al, 2020).However, the role of GDF15 in LUAD ferroptosis has not yet been reported and needs to be further elucidated.

HNF4A is a nuclear transcription factor, which is highly expressed in most cancers and is significantly associated with poor prognosis. Moreover, HNF4A is reported to play a role in ferroptosis (Dai, C., et al, 2020). Research has indicated that HNF4A expression is up-regulated in HCC(Dai, C., et al,2020), which can increase the synthesis of GSH and inhibit ferroptosis by up regulating the expression of STMN1 (a ferroptosis down-regulated factor) (Xu, L., et al, 2001).In lung cancer, activation of GSH biosynthesis-related genes can also lead to the inhibition of ferroptosis (Zhang, X., et al,2019).However, whether and how dysregulated expression of HNF4A regulates GSH production in LUAD remain to be explored. to ferroptosis, and ferroptosis in acute kidney injury may regulate inflammation by activating IL33. However, the relationship between ferroptosis in cancer and IL-33 is not clear.Previous studies have reported that CD8 + T cells induce ferroptosis of tumor cells in vivo(Wang, W., et al, 
279 2019; Tang, R., et al,2020) Our study also explored the relationship between risk score and 280 immune activity, but the mechanism between ferroptosis-related genes of LUAD and tumor 281 immunity needs to be further clarified.

282 The five genes included in our model play important roles in the occurrence and 283 development of LUAD, and most are related to ferroptosis in malignant tumors. Therefore, this 284 model could be a useful prognostic indicator of LUAD. However, our research has some 285 limitations that most bioinformatics analysis studies share. Firstly, all our data are from public 286 databases, so it will be necessary to verify the prognostic value of the model in clinical samples. 287 Secondly, this study failed to explore the underlying molecular mechanism of ferroptosis-related 288 genes in the occurrence and development of LUAD.

289 Conclusions

290 We developed a prognosis signature of five ferroptosis-related genes (ALOX15, DDIT4, 291 HNF4A, IL33, GDF15), which showed good reliability. Analysis of two independent databases 292 demonstrated that this model was independently related to OS in LUAD patients, and thus may 293 be a good predictor of LUAD prognosis.

294 Abbreviations

295 LUAD Lung adenocarcinoma;

296 UCSC The University of California, Santa Cruz

297 TCGA The Cancer Genome Atlas;

298 GEO Gene Expression Omnibus

299 DEGs Differentially expressed genes

300 OS Overall survival

301 ROC Receiver operating characteristic

302 GO Gene Ontology

303 KEGG Kyoto Encyclopedia of Genes and Genomes

304 SsGSEA Single-sample gene set enrichmentanalysis

305 3D PCA Principal component analysis 
306 HR Hazard ratio

307 CI Confidence interval

308 DCs Dendritic Cells

309 K-M Kaplan-Meier

310 ALOX15 The five screened genes were Arachidonate 15-Lipoxygenase

311 DDIT4 DNA Damage Inducible Transcript 4

312 HNF4A Hepatocyte Nuclear Factor 4Alpha

313 IL33 Interleukin 33

314 GDF15 Growth Differentiation Factor

\section{References}

316 Siegel, R.L., K.D. Miller, and A. Jemal, Cancer statistics, 2020. CA Cancer J Clin, 2020. 70(1):

317 p. 7-30.

318 Testa, U., G. Castelli, and E. Pelosi, Lung Cancers: Molecular Characterization, Clonal Heterogeneity and Evolution, and Cancer Stem Cells. Cancers (Basel), 2018. 10(8).

320

321

322

323

324

325

326

327

328

329

330

331

Eberhardt, W.E. and M. Stuschke, Multimodal treatment of non-small-cell lung cancer. Lancet, 2015. 386(9998): p. 1018-20.

Chen H, Carrot-Zhang J, Zhao Y, Hu H, Freeman SS, Yu S, Ha G, Taylor AM, Berger AC, Westlake L, Zheng Y, Zhang J, Ramachandran A, Zheng Q, Pan Y, Zheng D, Zheng S, Cheng C, Kuang M, Zhou X, Zhang Y, Li H, Ye T, Ma Y, Gao Z, Tao X, Han H, Shang J, Yu Y, Bao D, Huang Y, Li X, Zhang Y, Xiang J, Sun Y, Li Y, Cherniack AD, Campbell JD, Shi L, Meyerson M., Genomic and immune profiling of pre-invasive lung adenocarcinoma. Nat Commun, 2019. 10(1): p. 5472.

Latunde-Dada, G.O., Ferroptosis: Role of lipid peroxidation, iron and ferritinophagy. Biochim Biophys Acta Gen Subj, 2017. 1861(8): p. 1893-1900.

Stockwell BR, Friedmann Angeli JP, Bayir H, Bush AI, Conrad M, Dixon SJ, Fulda S, Gascón S, Hatzios SK, Kagan VE, Noel K, Jiang X, Linkermann A, Murphy ME, Overholtzer M, 
332

333

334

335

336

337

338

339

340

341

342

343

344

345

346

347

348

349

350

351

352

353

354

Oyagi A, Pagnussat GC, Park J, Ran Q, Rosenfeld CS, Salnikow K, Tang D, Torti FM, Torti SV, Toyokuni S, Woerpel KA, Zhang DD., Ferroptosis: A Regulated Cell Death Nexus Linking Metabolism, Redox Biology, and Disease. Cell, 2017. 171(2): p. 273-285.

Conrad M, Kagan VE, Bayir H, Pagnussat GC, Head B, Traber MG, Stockwell BR., Regulation of lipid peroxidation and ferroptosis in diverse species. Genes Dev, 2018. 32(9-10): p. 602-619.

Fearnhead, H.O., P. Vandenabeele, and T. Vanden Berghe, How do we fit ferroptosis in the family of regulated cell death? Cell Death Differ, 2017. 24(12): p. 1991-1998.

Liang C, Zhang X, Yang M, Dong X., Recent Progress in Ferroptosis Inducers for Cancer Therapy. Adv Mater, 2019. 31(51): p. e1904197.

Hassannia, B., P. Vandenabeele, and T. Vanden Berghe, Targeting Ferroptosis to Iron Out Cancer. Cancer Cell, 2019. 35(6): p. 830-849.

Dixon SJ, Lemberg KM, Lamprecht MR, Skouta R, Zaitsev EM, Gleason CE, Patel DN, Bauer AJ, Cantley AM, Yang WS, Morrison B 3rd, Stockwell BR., Ferroptosis: an irondependent form of nonapoptotic cell death[J].Cell,2012,149(5):1060-1072.

Wang SJ, Li D, Ou Y, Jiang L, Chen Y, Zhao Y, Gu W. , Acetylation is crucial for p53-mediated ferroptosis and tumor suppression[J].Cell Rep,2016,17(2):366-373.

Jiang L, Kon N, Li T, Wang SJ, Su T, Hibshoosh H, Baer R, Gu W., Ferroptosis as a p53mediated activity during tumour suppression[J].Nature,2015,520 (7545):57-62.

Ji X, Qian J, Rahman SMJ, Siska PJ, Zou Y, Harris BK, Hoeksema MD, Trenary IA, Heidi C, Eisenberg R, Rathmell JC, Young JD, Massion PP., xCT (SLC7A11)-mediated metabolic reprogramming promotes non-small cell lung cancer progression. Oncogene, 2018. 37(36): p. 5007-5019. 
355 Huang Y, Dai Z, Barbacioru C, Sadée W., Cystine-glutamate transporter SLC7A11 in cancer 356 chemosensitivity and chemoresistance. Cancer Res, 2005. 65(16): p. 7446-54.

357 Lai Y, Zhang Z, Li J, Li W, Huang Z, Zhang C, Li X, Zhao J., STYK1/NOK correlates with 358 ferroptosis in non-small cell lung carcinoma. Biochem Biophys Res Commun, 2019. 359 519(4): p. 659-666.

360 361

362 363

364

365 366

367

368 369

370

371

372

373 374

375

376

Guo J, Xu B, Han Q, Zhou H, Xia Y, Gong C, Dai X, Li Z, Wu G., Ferroptosis: A Novel Antitumor Action for Cisplatin. Cancer Res Treat, 2018. 50(2): p. 445-460.

Lachaier E, Louandre C, Godin C, Saidak Z, Baert M, Diouf M, Chauffert B, Galmiche A. , Sorafenib induces ferroptosis in human cancer cell lines originating from different solid tumors. Anticancer Res, 2014. 34(11): p. 6417-22.

Hangauer MJ, Viswanathan VS, Ryan MJ, Bole D, Eaton JK, Matov A, Galeas J, Dhruv HD, Berens ME, Schreiber SL, McCormick F, McManus MT., Drug-tolerant persister cancer cells are vulnerable to GPX4 inhibition[J].Nature,2017,551(7679):247-250.

Zhou, N. and J. Bao, FerrDb: a manually curated resource for regulators and markers of ferroptosis and ferroptosis-disease associations. Database (Oxford), 2020. 2020.

Ritchie ME, Phipson B, Wu D, Hu Y, Law CW, Shi W, Smyth GK., limma powers differential expression analyses for RNA-sequencing and microarray studies. Nucleic Acids Res. 2015 Apr 20;43(7):e47.

Szklarczyk D, Gable AL, Lyon D, Junge A, Wyder S, Huerta-Cepas J, Simonovic M, Doncheva NT, Morris JH, Bork P, Jensen LJ, Mering CV., STRING v11: protein-protein association networks with increased coverage, supporting functional discovery in genome-wide experimental datasets. Nucleic Acids Res, 2019. 47(D1): p. D607-d613.

Peer] reviewing PDF | (2021:01:57227:1:2:NEW 17 Jun 2021) 
377 Smoot ME, Ono K, Ruscheinski J, Wang PL, Ideker T., Cytoscape 2.8: new features for data 378 integration and network visualization. Bioinformatics, 2011. 27(3): p. 431-2.

379 Ranstam J, Cook JA. Kaplan-Meier curve. Br J Surg. 2017 Mar;104(4):442.

380 Ashburner M, Ball CA, Blake JA, Botstein D, Butler H, Cherry JM, Davis AP, Dolinski K, 381 Dwight SS, Eppig JT, Harris MA, Hill DP, Issel-Tarver L, Kasarskis A, Lewis S, Matese JC, Richardson JE, Ringwald M, Rubin GM, Sherlock G. , Gene ontology: tool for the 383 unification of biology. The Gene Ontology Consortium. Nat Genet. 2000 May;25(1):25-9.

384 385 386 387 388 389 390 391 392 393 394 395 396 397 398
Hänzelmann S, Castelo R, Guinney J., GSVA: gene set variation analysis for microarray and RNA-seq data. BMC Bioinformatics. 2013 Jan 16;14:7.

Diboun I, Wernisch L, Orengo CA, Koltzenburg M., Microarray analysis after RNA amplification can detect pronounced differences in gene expression using limma. BMC Genomics, 2006. 7: p. 252.

https://www.iarc.fr/faq/latest-global-cancer-data-2020-qa/.

Chen J, Yang H, Teo ASM, Amer LB, Sherbaf FG, Tan CQ, Alvarez JJS, Lu B, Lim JQ, Takano A, Nahar R, Lee YY, Phua CZJ, Chua KP, Suteja L, Chen PJ, Chang MM, Koh TPT, Ong BH, Anantham D, Hsu AAL, Gogna A, Too CW, Aung ZW, Lee YF, Wang L, Lim TKH, Wilm A, Choi PS, Ng PY, Toh CK, Lim WT, Ma S, Lim B, Liu J, Tam WL, Skanderup AJ, Yeong JPS, Tan EH, Creasy CL, Tan DSW, Hillmer AM, Zhai W. Genomic landscape of lung adenocarcinoma in East Asians. Nat Genet, 2020. 52(2): p. 177-186.

Huang, C.C., M. Du, and L. Wang, Bioinformatics Analysis for Circulating Cell-Free DNA in Cancer. Cancers (Basel), 2019. 11(6). 
399 Liang JY, Wang DS, Lin HC, Chen XX, Yang H, Zheng Y, Li YH. A Novel Ferroptosis-related 400 Gene Signature for Overall Survival Prediction in Patients with Hepatocellular

401

402

403

404

405

406

407

408

409

410

411

412

413

414

415

416

417

418

419

420

421

Carcinoma. Int J Biol Sci, 2020. 16(13): p. 2430-2441.

Dixon SJ, Lemberg KM, Lamprecht MR, Skouta R, Zaitsev EM, Gleason CE, Patel DN, Bauer AJ, Cantley AM, Yang WS, Morrison B 3rd, Stockwell BR. Ferroptosis: an irondependent form of nonapoptotic cell death. Cell, 2012. 149(5): p. 1060-72.

Stoyanovsky DA, Tyurina YY, Shrivastava I, Bahar I, Tyurin VA, Protchenko O, Jadhav S, Bolevich SB, Kozlov AV, Vladimirov YA, Shvedova AA, Philpott CC, Bayir H, Kagan VE. Iron catalysis of lipid peroxidation in ferroptosis: Regulated enzymatic or random free radical reaction? Free Radic Biol Med, 2019. 133: p. 153-161.

Probst L, Dächert J, Schenk B, Fulda S. Lipoxygenase inhibitors protect acute lymphoblastic leukemia cells from ferroptotic cell death. Biochem Pharmacol, 2017. 140: p. 41-52.

Wenzel SE, Tyurina YY, Zhao J, St Croix CM, Dar HH, Mao G, Tyurin VA, Anthonymuthu TS, Kapralov AA, Amoscato AA, Mikulska-Ruminska K, Shrivastava IH, Kenny EM, Yang Q, Rosenbaum JC, Sparvero LJ, Emlet DR, Wen X, Minami Y, Qu F, Watkins SC, Holman TR, VanDemark AP, Kellum JA, Bahar I, Bayır H, Kagan VE. PEBP1 Wardens Ferroptosis by Enabling Lipoxygenase Generation of Lipid Death Signals. Cell, 2017. 171(3): p. 628-641.e26.

Shureiqi I, Chen D, Lee JJ, Yang P, Newman RA, Brenner DE, Lotan R, Fischer SM, Lippman SM. 15-LOX-1: a novel molecular target of nonsteroidal anti-inflammatory drug-induced apoptosis in colorectal cancer cells. J Natl Cancer Inst, 2000. 92(14): p. 1136-42.

Tang S, Bhatia B, Maldonado CJ, Yang P, Newman RA, Liu J, Chandra D, Traag J, Klein RD, Fischer SM, Chopra D, Shen J, Zhau HE, Chung LW, Tang DG.Evidence that 
422

423

424 425

426

427 428

arachidonate 15-lipoxygenase 2 is a negative cell cycle regulator in normal prostate epithelial cells. J Biol Chem, 2002. 277(18): p. 16189-201.

Jiang, W.G., A. Douglas-Jones, and R.E. Mansel, Levels of expression of lipoxygenases and cyclooxygenase-2 in human breast cancer. Prostaglandins Leukot Essent Fatty Acids, 2003. 69(4): p. 275-81.

Gonzalez AL, Roberts RL, Massion PP, Olson SJ, Shyr Y, Shappell SB. 15-Lipoxygenase-2 expression in benign and neoplastic lung: an immunohistochemical study and correlation with tumor grade and proliferation. Hum Pathol, 2004. 35(7): p. 840-9.

Shintoku R, Takigawa Y, Yamada K, Kubota C, Yoshimoto Y, Takeuchi T, Koshiishi I, Torii S, Lipoxygenase-mediated generation of lipid peroxides enhances ferroptosis induced by erastin and RSL3. Cancer Sci, 2017. 108(11): p. 2187-2194.

Smith, E.R. and X.X. Xu, REDD1, a new Ras oncogenic effector. Cell Cycle, 2009. 8(5): p. 6756.

Tirado-Hurtado, I., W. Fajardo, and J.A. Pinto, DNA Damage Inducible Transcript 4 Gene: The Switch of the Metabolism as Potential Target in Cancer. Front Oncol, 2018. 8: p. 106.

Jin HO, Hong SE, Kim JY, Kim MR, Chang YH, Hong YJ, Lee JK, Park IC, Induction of HSP27 and HSP70 by constitutive overexpression of Redd1 confers resistance of lung cancer cells to ionizing radiation. Oncol Rep, 2019. 41(5): p. 3119-3126.

Lu X, He X, Su J, Wang J, Liu X, Xu K, De W, Zhang E, Guo R, Shi YE, EZH2-Mediated Epigenetic Suppression of GDF15 Predicts a Poor Prognosis and Regulates Cell Proliferation in Non-Small-Cell Lung Cancer. Mol Ther Nucleic Acids, 2018. 12: p. 309318. 
444 Duan L, Pang HL, Chen WJ, Shen WW, Cao PP, Wang SM, Liu LL, Zhang HL., The role of 445 GDF15 in bone metastasis of lung adenocarcinoma cells. Oncol Rep, 2019. 41(4): p. $446 \quad 2379-2388$.

447 Chen L, Qiao L, Bian Y, Sun X., GDF15 knockdown promotes erastin-induced ferroptosis by 448 449 decreasing SLC7A11 expression. Biochem Biophys Res Commun, 2020. 526(2): p. 293-

450

451

452

453

454

455

456

457

458

459

460

461

462

463

464

465

466 299.

Dai C, Chen X, Li J, Comish P, Kang R, Tang D. Transcription factors in ferroptotic cell death. Cancer Gene Ther. 2020 Sep;27(9):645-656.

Xu L, Hui L, Wang S, Gong J, Jin Y, Wang Y, Ji Y, Wu X, Han Z, Hu G. Expression profiling suggested a regulatory role of liver-enriched transcription factors in human hepatocellular carcinoma. Cancer Res. 2001 Apr 1;61(7):3176-81.

Zhang X, Du L, Qiao Y, Zhang X, Zheng W, Wu Q, Chen Y, Zhu G, Liu Y, Bian Z, Guo S, Yang Y, Ma L, Yu Y, Pan Q, Sun F, Wang J. Ferroptosis is governed by differential regulation of transcription in liver cancer. Redox Biol. 2019 Jun;24:101211.

Alvarez SW, Sviderskiy VO, Terzi EM, Papagiannakopoulos T, Moreira AL, Adams S, Sabatini DM, Birsoy K, Possemato R. NFS1 undergoes positive selection in lung tumours and protects cells from ferroptosis. Nature. 2017 Nov 30;551(7682):639-643.

Martin-Sanchez D, Ruiz-Andres O, Poveda J, Carrasco S, Cannata-Ortiz P, Sanchez-Niño MD, Ruiz Ortega M, Egido J, Linkermann A, Ortiz A, Sanz AB. Ferroptosis, but Not Necroptosis, Is Important in Nephrotoxic Folic Acid-Induced AKI. J Am Soc Nephrol. 2017 Jan;28(1):218-229.

Wang W, Green M, Choi JE, Gijón M, Kennedy PD, Johnson JK, Liao P, Lang X, Kryczek I, Sell A, Xia H, Zhou J, Li G, Li J, Li W, Wei S, Vatan L, Zhang H, Szeliga W, Gu W, Liu 
467 R, Lawrence TS, Lamb C, Tanno Y, Cieslik M, Stone E, Georgiou G, Chan TA, 468 Chinnaiyan A, Zou W. CD8+ T cells regulate tumour ferroptosis during cancer 469 immunotherapy. Nature. 2019 May;569(7755):270-274.

470 Tang R, Xu J, Zhang B, Liu J, Liang C, Hua J, Meng Q, Yu X, Shi S. Ferroptosis, necroptosis, 471 and pyroptosis in anticancer immunity. J Hematol Oncol. 2020 Aug 10;13(1):110. 
Figure 1

Figure 1. Flow diagram of the bioinformatic analysis in this study

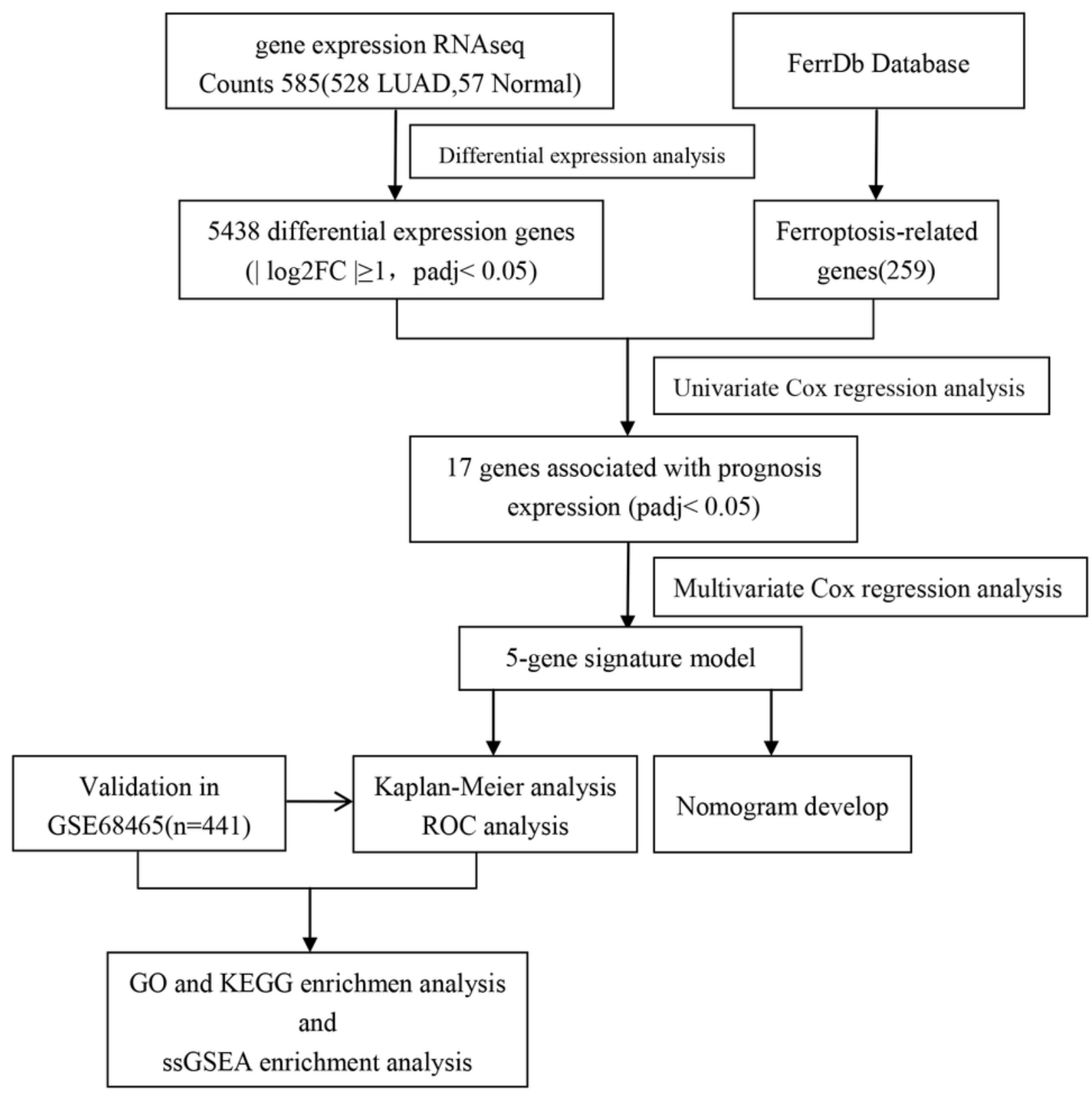


Figure 2

Figure 2. The differentially ferroptosis-related genes of LUAD in the TCGA database and FerrDb database.

a

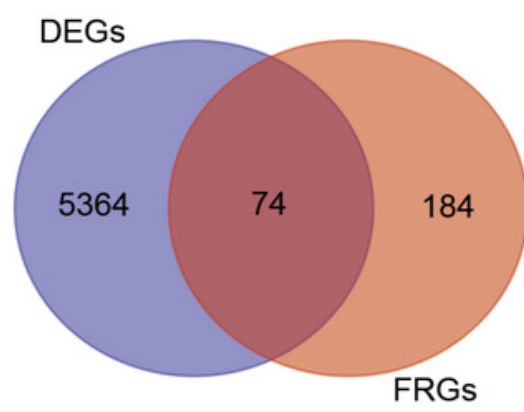

b

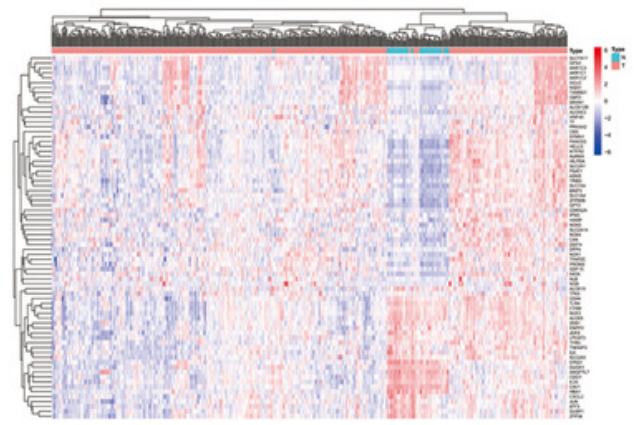

C

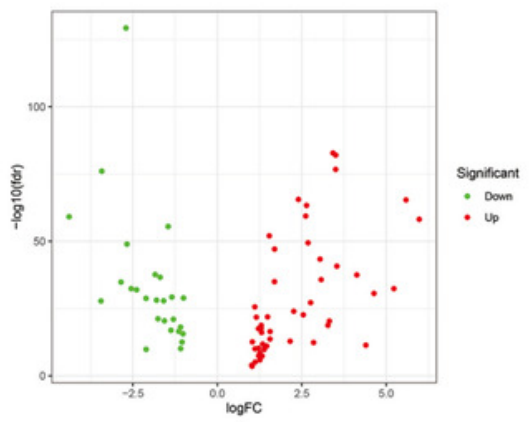


Figure 3

Figure 2. The differentially ferroptosis-related genes of LUAD in the TCGA database and FerrDb database.

a

$\begin{array}{lrr}\text { MTFR2 } & \text { pvalue } & \begin{array}{r}\text { Hazard ratio } \\ \text { GCLC }\end{array} \\ \text { TXNRD1 } & 0.014 & 1.140(1.027-1.266) \\ \text { ALOX15 } & 0.040 & 1.086(1.004-1.176) \\ \text { DDIT4 } & 0.022 & 1.107(1.015-1.208) \\ \text { CDO1 } & 0.004 & 0.917(0.866-0.972) \\ \text { HNF4A } & 0.008 & 1.155(1.038-1.285) \\ \text { IL33 } & 0.034 & 0.897(0.811-0.992) \\ \text { GDF15 } & 0.026 & 1.055(1.006-1.107) \\ \text { AURKA } & 0.004 & 0.872(0.794-0.958) \\ \text { SLC7A11 } & 0.013 & 0.906(0.839-0.979) \\ \text { SLC2A1 } & 0.004 & 1.170(1.050-1.303) \\ \text { ALOXE3 } & 0.038 & 1.074(1.004-1.149) \\ \text { DPP4 } & <0.001 & 1.180(1.078-1.292) \\ \text { ANGPTL7 } & 0.021 & 1.105(1.015-1.202) \\ \text { DUOX1 } & 0.037 & 0.930(0.868-0.996) \\ \text { SLC7A5 } & 0.012 & 0.891(0.815-0.975) \\ & 0.033 & 0.919(0.850-0.993) \\ & 0.030 & 1.119(1.011-1.239)\end{array}$

1.119(1.011-1.239)

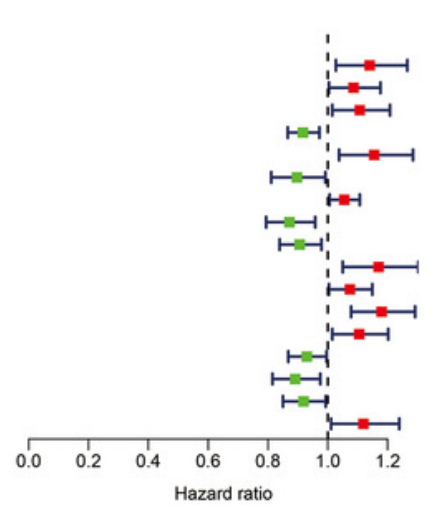

d
C

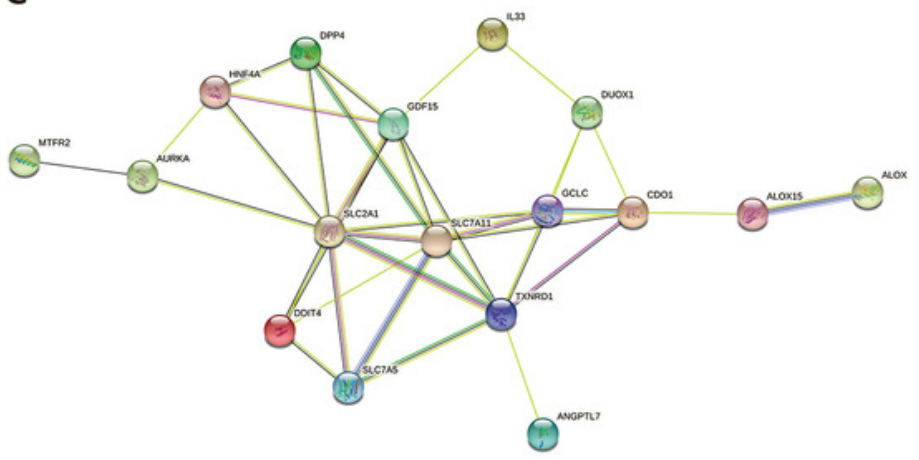

b

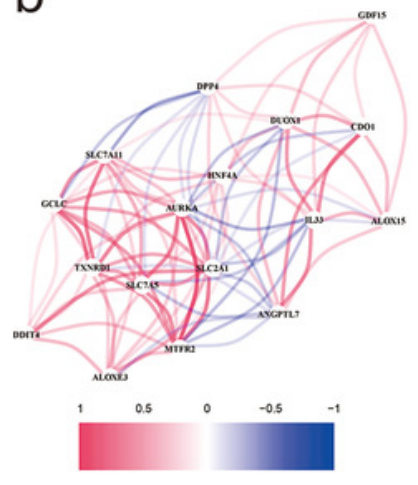

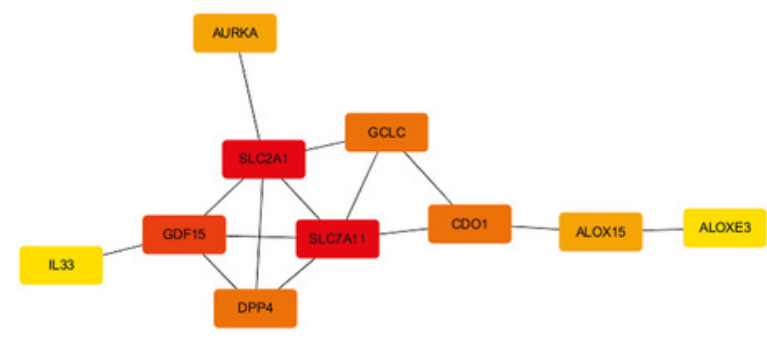


Figure 4

Fig.4 Forest plot of multivariate Cox proportional hazards regression analysis of overall survival for 5-gene signature model in the TCGA cohort.

\section{Hazard ratio}

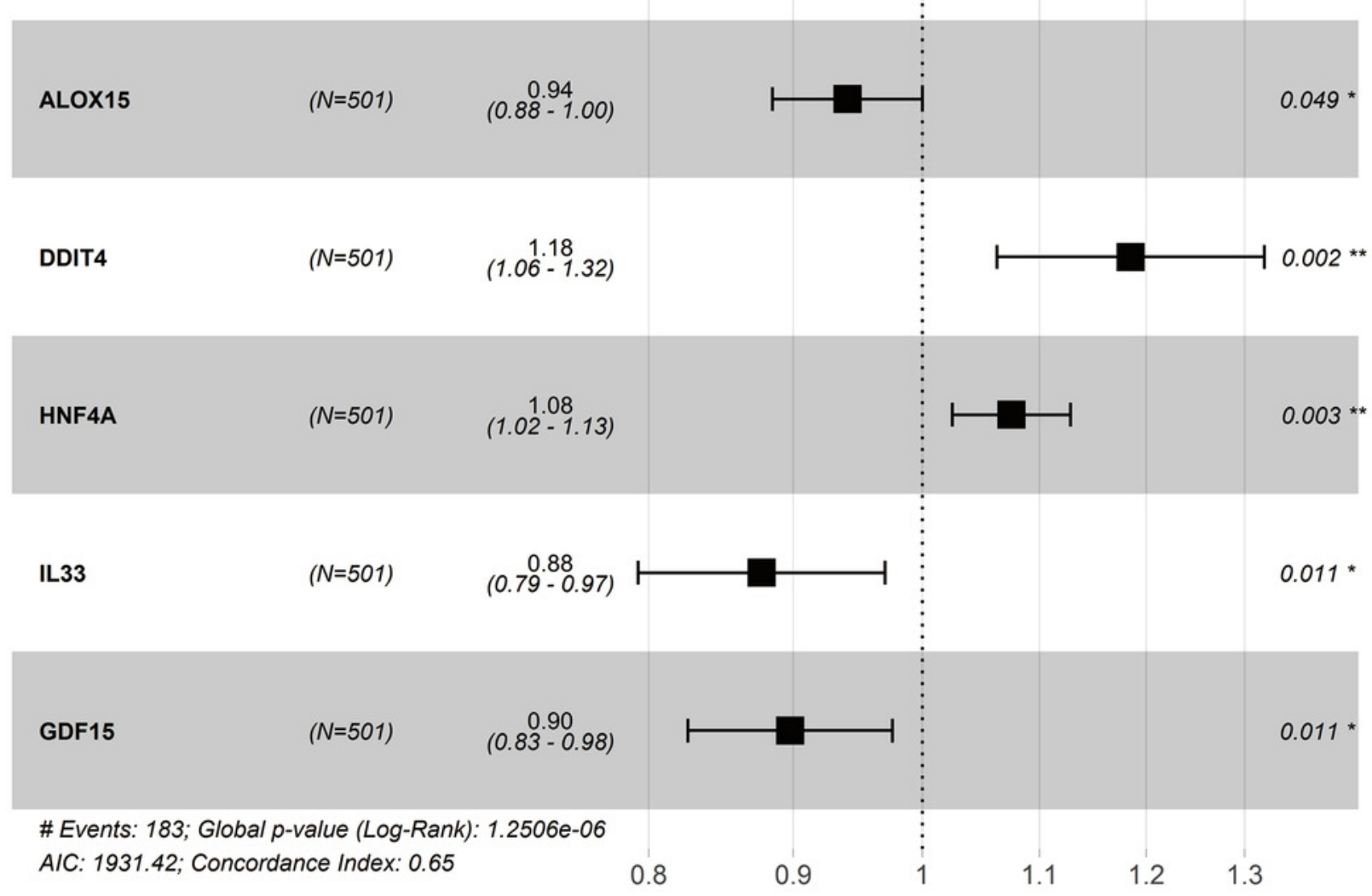


Figure 5

Figure 5. Construction and predictive accuracy in different risk models with TCGA and GSE68465 dataset. 

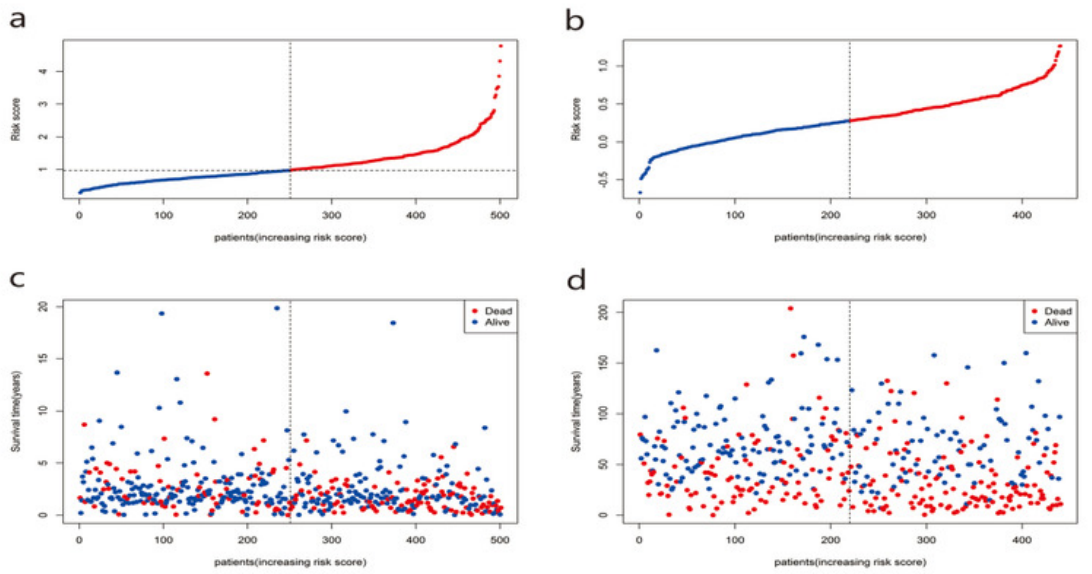

e

f
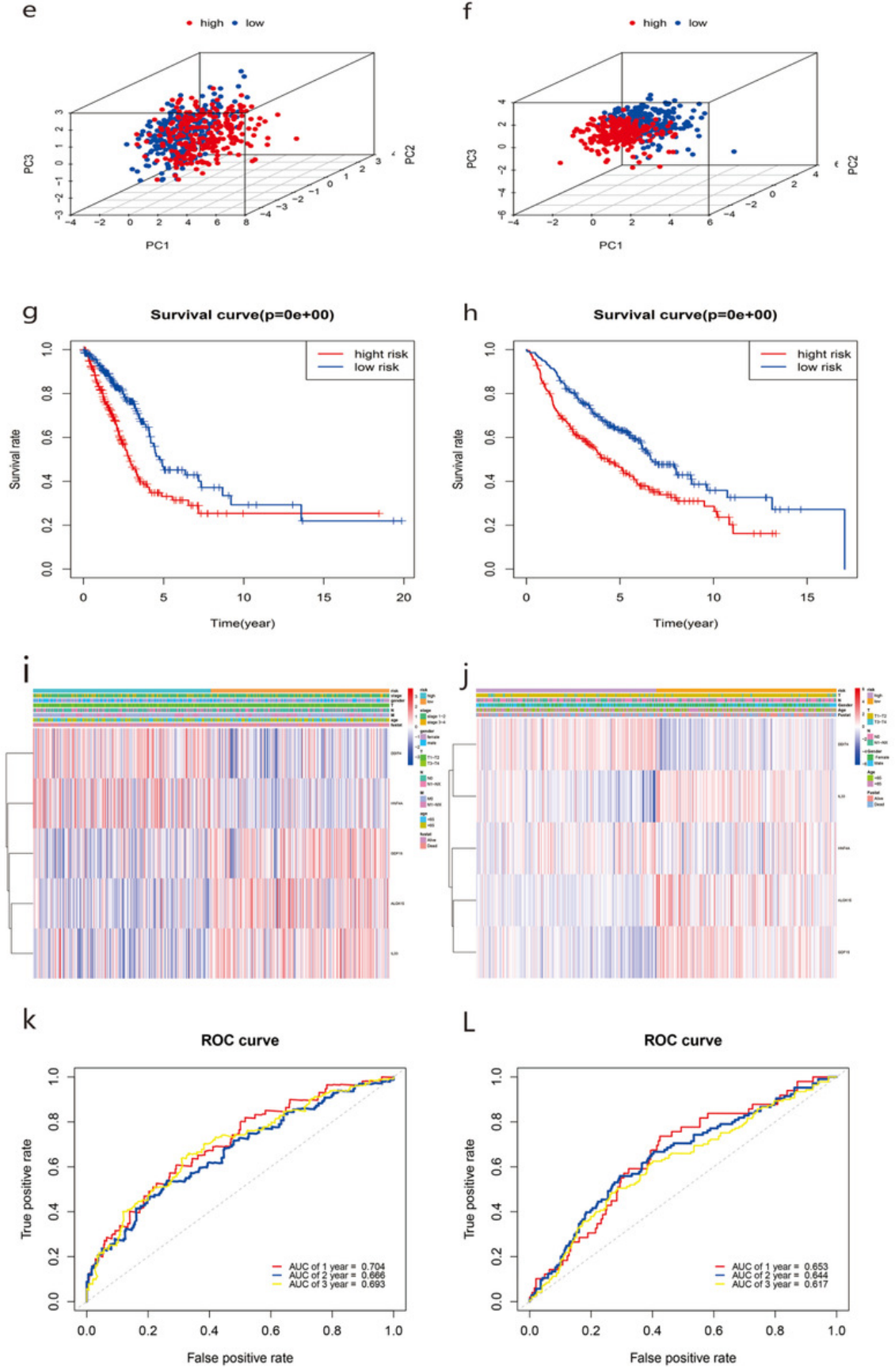
Figure 6

Figure 6. Construction of survival prediction nomogram and calibration plot of the nomogram.
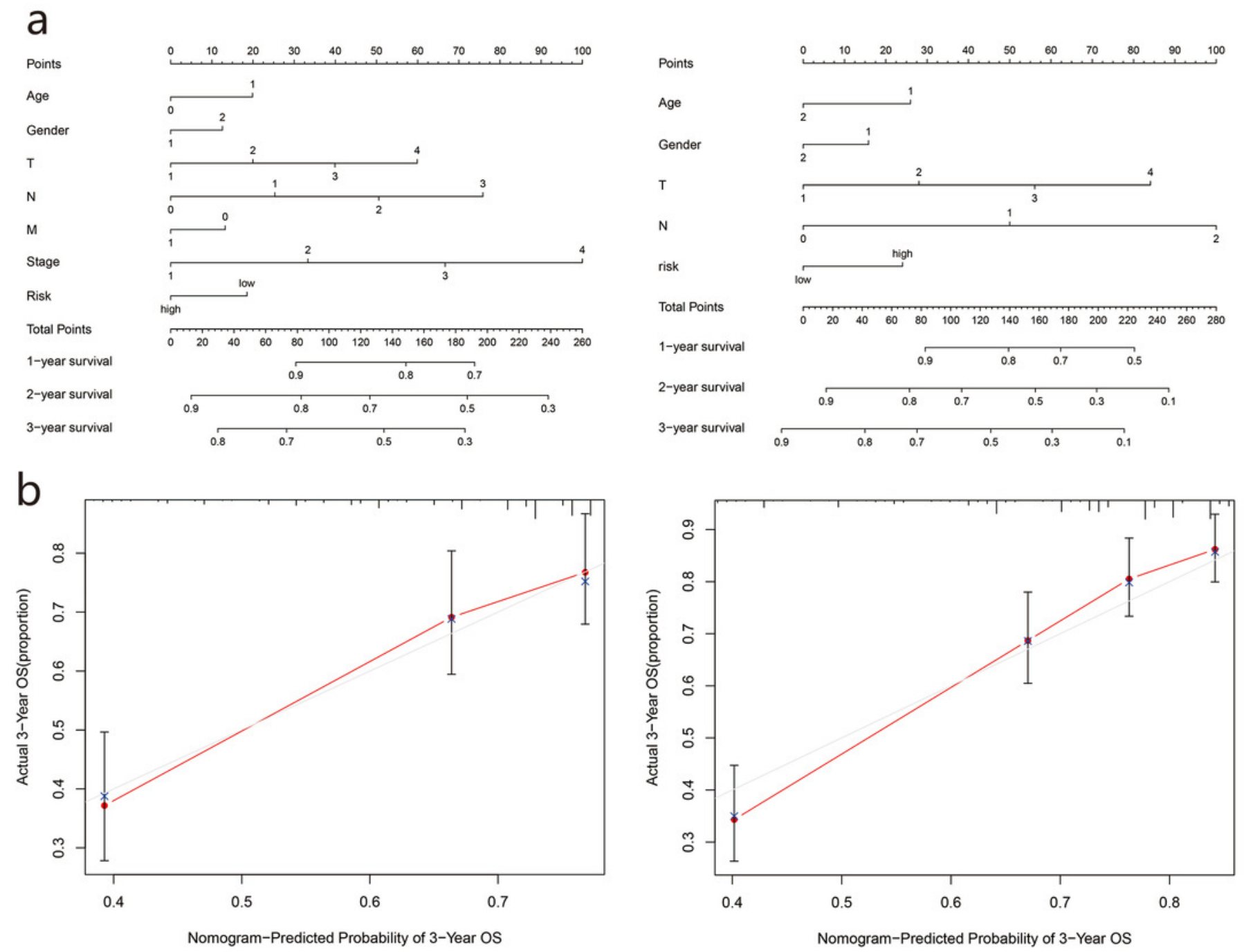
Figure 7

Figure 7. Forest plot of univariate and multivariate Cox regression analysis in TCGA set (a) and GSE68465 validation set $\square \mathrm{b} \square$.
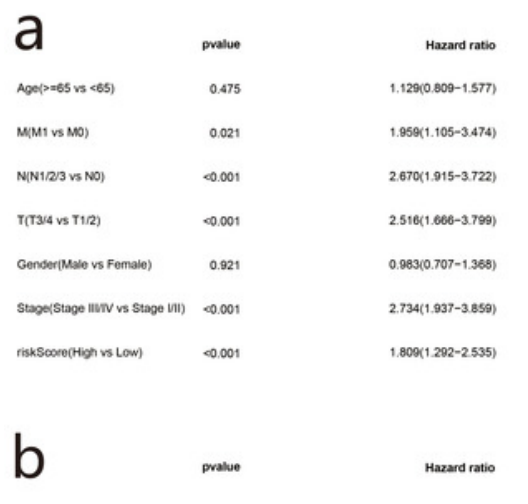

AOe( $\times 065$ vs $<65) \quad 0.014$

Genser(Maic us Female) $\quad 0.007$

N(N1/2 vs NO) $\quad \infty .001$

$T(T 3 / 4$ vs T1/2) $\quad \infty .001$

risksocre(High us Low) $\quad 0.001$

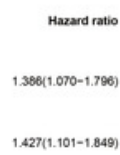

1.427(1.101-1.849)

2.768(2. 134-3.590)

2.772(1.914-4.016)

$1.625(1.254-2.105)$
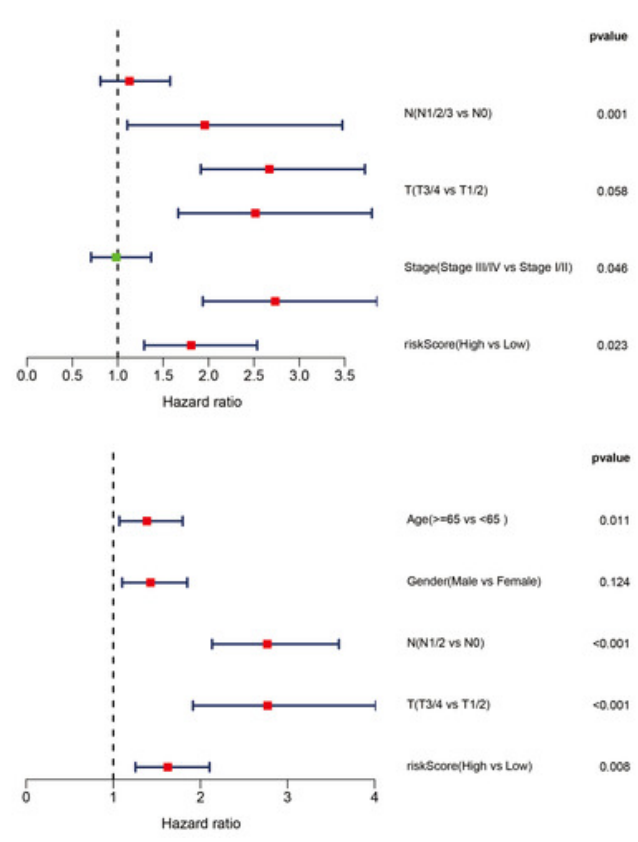
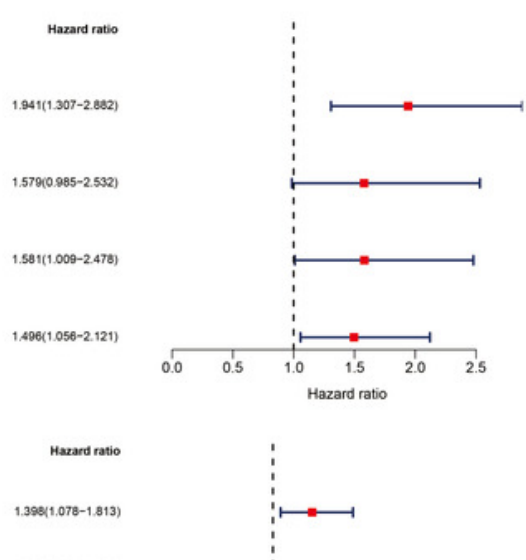

1.2320.0.945-1.606)

2694(2.073-3.501)

2.438(1.668-3.556)

$1.425(1.098-1.850)$

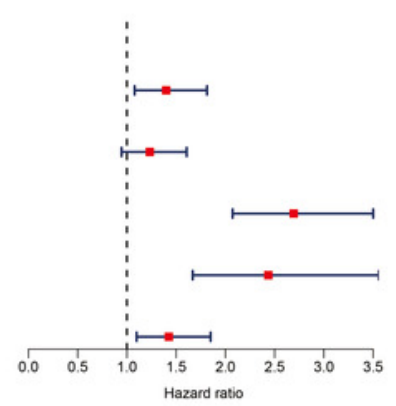


Figure 8

Figure 8. Functional enrichment analysis of two risk groups by Gene Ontology (GO) and Kyoto Encyclopedia of genes and genomes (KEGG) 
a

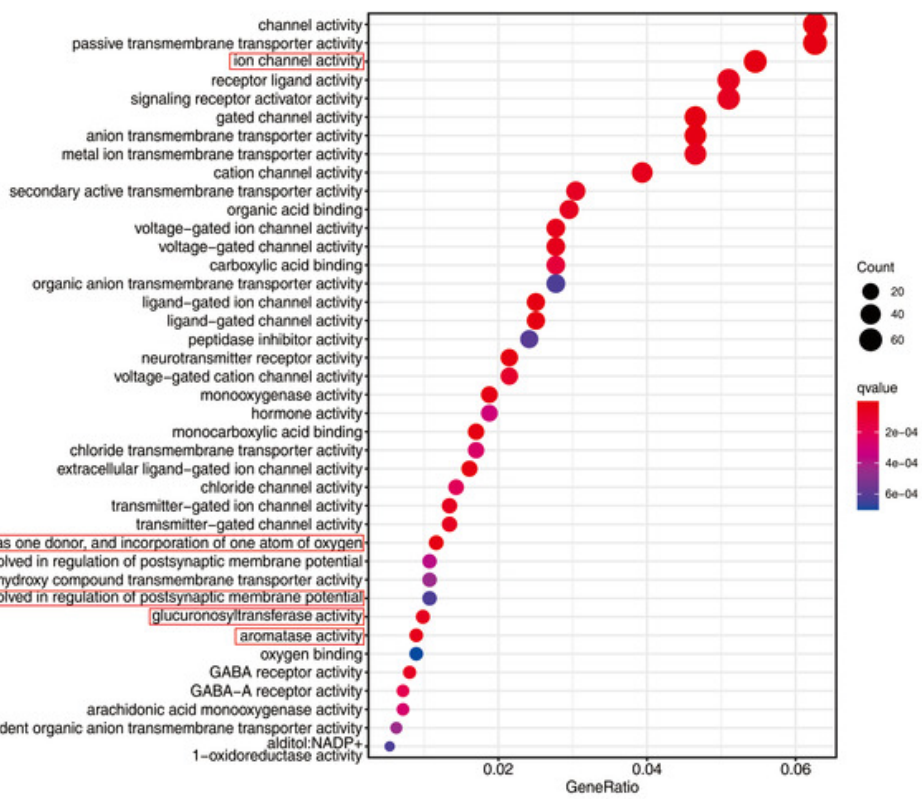

b
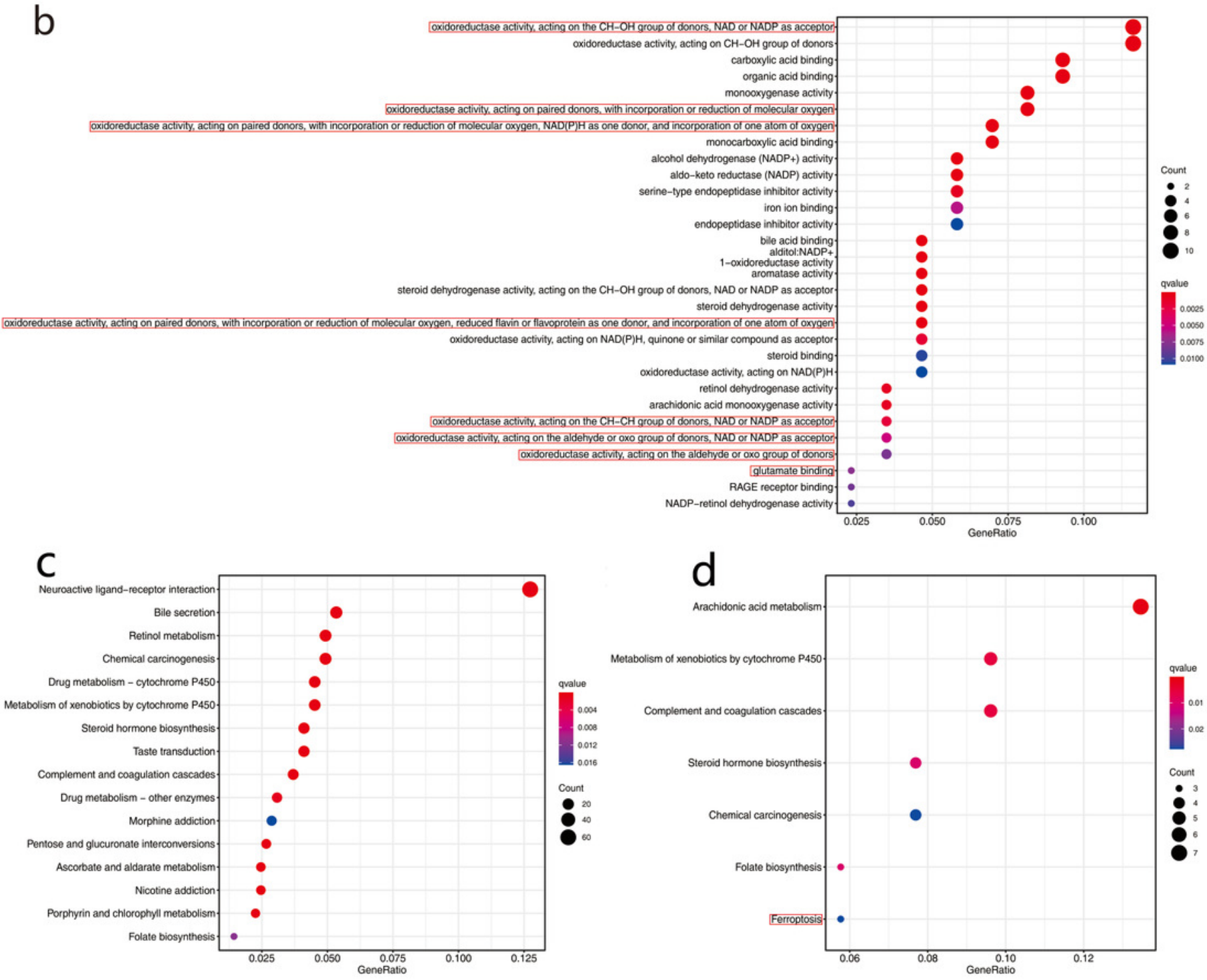
Figure 9

Figure 9. Comparison of the sSGSEA scores between two risk groups
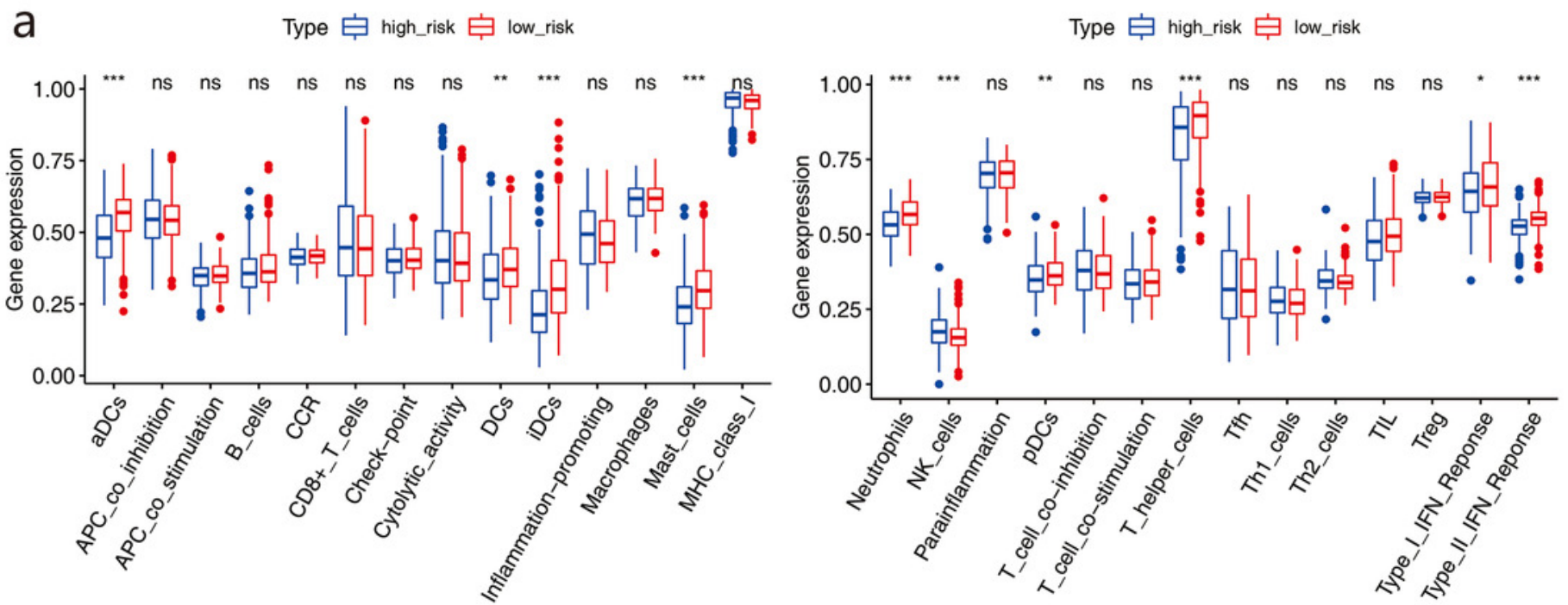

b

Type 追 High_risk 追 low_risk

Type 追 High_risk 追 low_risk
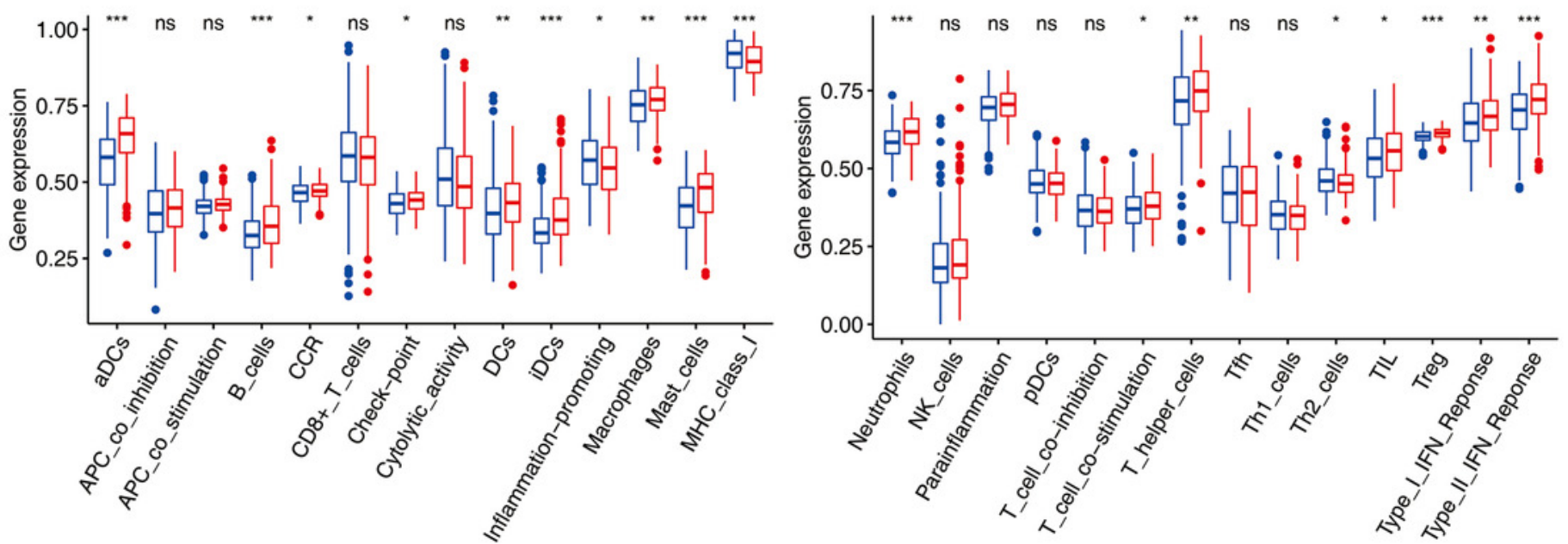


\section{Table $\mathbf{1}$ (on next page)}

Table.1 Clinicopathological characteristics of the LUAD patients 
1 Table.1 Clinicopathological characteristics of the LUAD patients

\begin{tabular}{|c|c|c|c|c|}
\hline \multirow{2}{*}{ Characteristics } & \multicolumn{2}{|c|}{ TCGA $(n=501)$} & \multicolumn{2}{|c|}{$\operatorname{GSE68465}(n=441)$} \\
\hline & Number of cases & $\%$ & Number of cases & $\%$ \\
\hline \multicolumn{5}{|l|}{ Age (years) } \\
\hline$\geq 65$ & 270 & 53.9 & 229 & 51.9 \\
\hline$<65$ & 231 & 46.1 & 212 & 48.1 \\
\hline \multicolumn{5}{|l|}{ Gender } \\
\hline Male & 232 & 46.3 & 222 & 50.3 \\
\hline Female & 269 & 53.7 & 219 & 49.7 \\
\hline \multicolumn{5}{|l|}{$\mathrm{T}$} \\
\hline $\mathrm{T} 1-2$ & 436 & 87 & 401 & 90.9 \\
\hline T3-4 & 65 & 13 & 40 & 9.1 \\
\hline \multicolumn{5}{|l|}{$\mathrm{N}$} \\
\hline No & 329 & 65.7 & 299 & 59.7 \\
\hline $\mathrm{N} 1-\mathrm{Nx}$ & 172 & 34.3 & 142 & 40.3 \\
\hline \multicolumn{5}{|l|}{$\mathrm{M}$} \\
\hline M0 & 336 & 67.1 & NA & NA \\
\hline M1-Mx & 165 & 32.9 & NA & NA \\
\hline \multicolumn{5}{|l|}{ Stage } \\
\hline Stage 1-2 & 394 & 78.6 & NA & NA \\
\hline Stage 3-4 & 107 & 21.4 & NA & NA \\
\hline
\end{tabular}


Table 2 (on next page)

Table2. The prognostic value of five ferroptosis-related genes 
2 Table2.

The

\begin{tabular}{cccccc}
\hline Gene & Coef & HR & HR.95L & HR.95H & P vaule \\
\hline ALOX15 & -0.061165052 & 0.940667967 & 0.88 & 1.00 & 0.049434651 \\
DDIT4 & 0.169594473 & 1.184824275 & 1.06 & 1.32 & 0.002251552 \\
HNF4A & 0.072356312 & 1.075038325 & 1.02 & 1.13 & 0.003200809 \\
IL33 & -0.131023567 & 0.877197101 & 0.79 & 0.97 & 0.010695027 \\
& & & & & \\
GDF15 & -0.107882858 & 0.897732752 & 0.83 & 0.98 & 0.011022652 \\
\hline
\end{tabular}

3 prognostic value of five ferroptosis-related genes

4 Notes: Coef is the risk coefficient of each gene, if the value of coef $>0$, it is regarded as the risk factor of 5 prognosis, otherwise it is regarded as the protective factor of prognosis. HR, hazard ratio. 